\title{
Stable laser-ion acceleration in the light sail regime
}

\author{
S. Steinke, ${ }^{1, *}$ P. Hilz, ${ }^{2}$ M. Schnürer, ${ }^{1}$ G. Priebe, ${ }^{1}$ J. Bränzel, ${ }^{1}$ F. Abicht, ${ }^{1}$ D. Kiefer, ${ }^{2,3}$ C. Kreuzer, ${ }^{2}$ T. Ostermayr, ${ }^{2}$ \\ J. Schreiber, ${ }^{2,3}$ A. A. Andreev, ${ }^{1,4, \dagger}$ T. P. Yu, ${ }^{5,6,}$ A. Pukhov, ${ }^{5}$ and W. Sandner ${ }^{1}$ \\ ${ }^{1}$ Max-Born-Institut, D-12489 Berlin, Germany \\ ${ }^{2}$ Fakultät f. Physik, LMU München, D-85748, Garching, Germany \\ ${ }^{3}$ Max-Planck-Institut fur Quantenoptik, D-85748 Garching, Germany \\ ${ }^{4}$ Vavilov State Optical Institute, Sankt Petersburg 199034, Russia \\ ${ }^{5}$ Institut f. Theoretische Physik I, Heinrich-Heine-Universität Düsseldorf, Düsseldorf D-40225, Germany \\ ${ }^{6}$ Department of Physics, National University of Defense Technology, Changsha 410073, China
}

(Received 23 February 2012; published 30 January 2013)

\begin{abstract}
We present experimental results on ion acceleration with circularly polarized, ultrahigh contrast laser pulses focused to peak intensities of $5 \times 10^{19} \mathrm{~W} \mathrm{~cm}^{-2}$ onto polymer targets of a few 10 nanometer thickness. We observed spatially and energetically separated protons and carbon ions that accumulate to pronounced peaks around $2 \mathrm{MeV}$ containing as much as $6.5 \%$ of the laser energy. Based on particle-incell simulation, we illustrate that an early separation of heavier carbon ions and lighter protons creates a stable interface that is maintained beyond the end of the radiation pressure dominated acceleration process.
\end{abstract}

DOI: 10.1103/PhysRevSTAB.16.011303

Laser plasma ion acceleration from nanometer-scale solid density foil targets has gained substantial interest during the past years [1-6] since it is capable of generating monoenergetic ion bunches with high efficiency via novel acceleration mechanisms such as radiation pressure acceleration (RPA) [7-17]. Although barely competitive in average flux, the density of such laser accelerated ion bunches can reach almost solid density, exceeding conventional sources by many orders of magnitude, enabling the study of a manifold of nonlinear processes such as ion fast ignition or fission fusion processes. For RPA being the dominant mechanism at laser intensities below $10^{23} \mathrm{~W} \mathrm{~cm}^{-2}$ it is necessary to suppress electron heating and, consequently, expansion of the target. At laser intensities of $\sim 10^{19} \mathrm{~W} \mathrm{~cm}^{-2}$ routinely realized these days, electrons can be compressed to a highly dense layer by using circularly polarized light $[10,11]$. The electric field set up by the laser induced charge separation of electrons and ions initially at rest is of the order of the laser field, i.e. $\sim 10^{13} \mathrm{~V} / \mathrm{m}$, and sustained for the duration of the laser pulse, typically a few tens of fs. In a foil with a thickness of $25 \mathrm{~nm}$ that contains two ion species, a double layer is formed after a few laser cycles. Rayleigh-Taylor-like instabilities are buffered in the layer of the heavier ions while the acceleration of

\footnotetext{
*sven.steinke@mbi-berlin.de

andreev@mbi-berlin.de

*tongpu@nudt.edu.cn
}

Published by the American Physical Society under the terms of the Creative Commons Attribution 3.0 License. Further distribution of this work must maintain attribution to the author(s) and the published article's title, journal citation, and DOI.
PACS numbers: 52.38.Kd, 41.75.Jv, 52.50.Jm, 52.65.Rr

the lighter ion species is stabilized [16], regardless of the tremendous acceleration $\left(\sim 10^{21} \mathrm{~ms}^{-2}\right)$ to which they are exposed. To achieve the highest possible compression, a pressure balance has to be established: The driving radiation pressure has to be equalized by the restoring electrostatic pressure due to the charge separation of electrons and ions. Subsequently, the laser energy is transferred to the accelerated plasma (light sail) by the relativistic Doppler effect.

We present the first experimental investigation of this stable RPA regime utilizing polymer foils with a high hydrogen content, irradiated by a circularly polarized laser pulse. Freestanding polyvinyl formal (Formvar) films with thicknesses down to $15 \mathrm{~nm}$ have been illuminated at normal incidence and ultrahigh temporal laser pulse contrast. In the experiments, the early separation of heavier carbon ions and lighter protons is expressed by the observation of isolated energy distributions of the two species. Around an optimum target thickness that depends on laser intensity, the fastest carbon ions coincide with the slowest protons, both accumulate to slightly separated, distinct maxima. These spectral characteristics are observed for a variety of target thicknesses and laser pulse durations. At the optimum thickness, the measured velocity of the ion interface has a maximum and is in excellent agreement with the analytical 1D light sail model. Our interpretation is supported by both 2D and 3D particle-in-cell (PIC) simulations.

The experiments were performed at the $35 \mathrm{TW}$ laser facility at the Max Born Institute in Berlin. The Ti: sapphire laser delivers pulses with an energy of $1.2 \mathrm{~J}$ in $34 \mathrm{fs}$ FWHM at a central wavelength of $\lambda_{0}=$ $810 \mathrm{~nm}$. The intrinsic amplified spontaneous emission 
contrast ratio of the laser equipped with a front end based on cross polarized wave (XPW) generation [18] was characterized to be smaller than $10^{-11}$ on a few picosecond scale [19]. This contrast was further enhanced by a recollimating double plasma mirror (DPM) $[20,21]$ to an estimated value of $\sim 10^{-15}$. Taking into account the $60 \%$ energy throughput of the DPM system, laser pulses with an energy of $E_{L}=0.7 \mathrm{~J}$ were focused to a focal spot of $3.6 \mu \mathrm{m}$ diameter (FWHM) using a $f / 2.5$ off-axis parabolic mirror resulting in a peak intensity for circular polarization of $I_{\text {peak }}=5 \times 10^{19} \mathrm{~W} \mathrm{~cm}^{-2}$ corresponding to a relativistically normalized vector potential of $a_{0} \approx 5$. A mica crystal $\lambda / 4$ wave plate was used to change the laser polarization to circular behind the plasma mirror setup. Freestanding Formvar films of thickness $15-50 \mathrm{~nm}$ and density $\rho=1.2 \mathrm{~g} \mathrm{~cm}^{-3}$ were irradiated at normal incidence in the focal plane of the driving laser. The thickness $l$ of the Formvar films was characterized by an atomic force microscope, including the water and hydrocarbon contamination [22] on the surfaces. Their chemical structure $\left(\mathrm{C}_{9} \mathrm{H}_{20} \mathrm{O}_{3}\right)$ was determined by elastic recoil detection analysis (ERDA). The resulting ion beams were analyzed with a Thomson Parabola spectrometer in laser propagation direction and visualized by a CCD camera coupled to a microchannel plate with phosphor screen [23]. Ion spectra are shown in Fig. 1 for laser pulse durations of 34 and $70 \mathrm{fs.}$ To compare the velocities of the individual ion species, the energy scale is given in $\mathrm{MeV}$ per atomic mass unit $u$. While the spectral shape of the protons exhibit maxima, the spectra of the fully ionized carbon consist of two parts: a low energy part and a peak at the high energy cutoff. In all cases the energy/u distributions suggest that the majority of protons travels faster than the carbon ions. With a laser pulse duration of $t=34 \mathrm{fs}$, an optimum ion acceleration was obtained at a target thickness of $l=(25 \pm 2) \mathrm{nm}$ with an energy of $1.8 \mathrm{MeV}$. The existence of such an optimum is understood by means of a 1D RPA model [8]. Solving the balance condition of laser radiation pressure and the electrostatic pressure and further assuming total reflectivity and a spatial Gaussian laser pulse shape, for the optimum thickness $l_{\text {opt }}$ gives $l_{\mathrm{opt}} \simeq \sqrt{2} a_{0} \lambda_{0}\left(n_{c} / n_{e}\right) \quad[24,25]$, where $a_{0}=e E_{0} / m_{e} c \omega$ and the critical plasma density $n_{c}=\epsilon_{0} m_{e} \omega^{2} / e^{2}$ with $m_{e}, n_{e}$ the electron mass and density, $c, \omega$, and $E_{0}$ the speed of light in vacuum, the laser frequency, and peak electric field, respectively. Assuming full ionization in the focal volume, the electron density becomes $n_{e} \simeq$ $240 n_{c}$ and hence, the optimum target thickness $l_{\mathrm{opt}} \simeq$ $24 \mathrm{~nm}$ in agreement with the experimentally obtained $l=(25 \pm 2) \mathrm{nm}$. The transition into an unstable regime is demonstrated by Fig. 1(a) where the target thickness is well below the optimum. Here, the interface breaks apart, i.e., carbon ions penetrate into the layer of protons and the spectra exhibit a high shot-to-shot fluctuation.

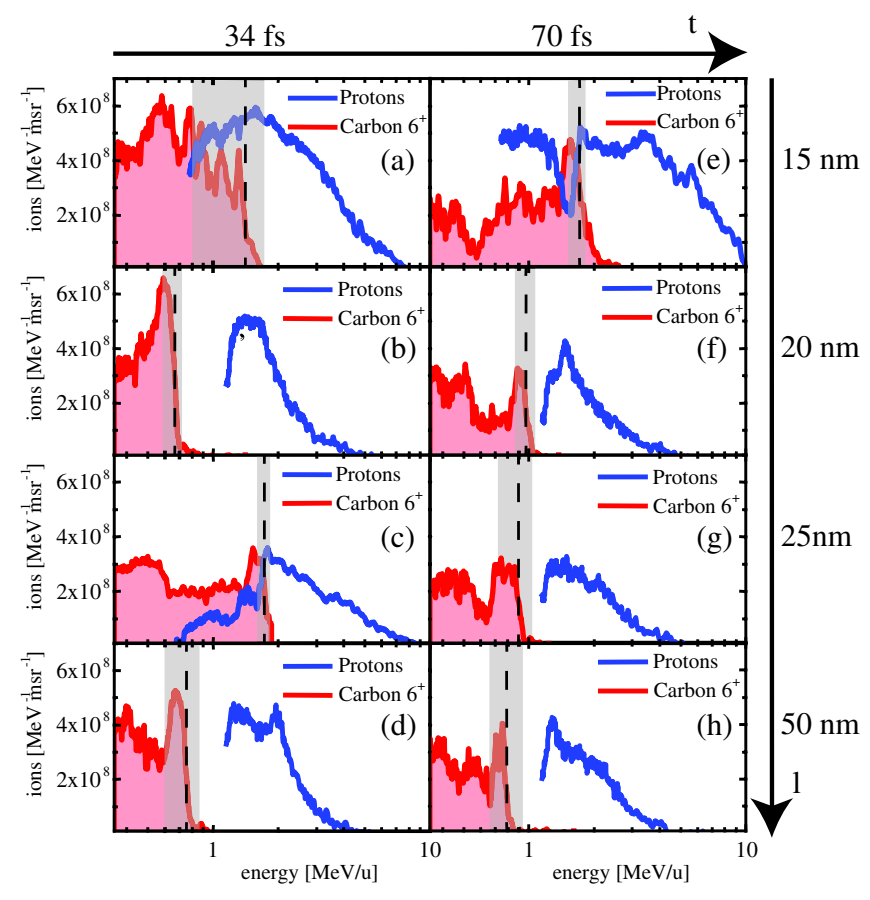

FIG. 1. Measured proton (blue) and $\mathrm{C}^{6+}$ (red) spectra generated using circularly polarized laser pulses with durations $34 \mathrm{fs}$ (a)-(d), $70 \mathrm{fs}$ (e)-(h), and target thicknesses of ranging from $l=$ $15 \mathrm{~nm}$ to $l=50 \mathrm{~nm}$. To compare the velocities of the individual ion species, the energy scale is given in $\mathrm{MeV}$ per atomic mass unit $u$, the ion interface is indicated as the $\mathrm{C}^{6+}$ cutoff (black dashed line) with its standard deviation of three laser shots (grey area). The low energy cutoff of the proton spectra is due to the detector edge which was modified in the case of (a), (c), and (e) to resolve the low energy part of the proton trace.

The interaction is dominated by Rayleigh-Taylor-like instabilities [26]. We investigated the balance condition further by performing the experiment with laser pulse duration of 70 fs, i.e. $a_{0} \approx 3.5$ [Figs. $1(\mathrm{e})-1(\mathrm{~h})$ ]. The optimum in terms of the highest interface velocity was obtained with targets of thickness $15 \pm 2 \mathrm{~nm}$, in good agreement with the $17 \mathrm{~nm}$ predicted by the model. After the RPA is initiated by such a pressure balance and, hence, the creation of a highly compressed electron layer [13], in the following the energy of the laser pulse is transferred to kinetic energy of the target by the Doppler redshift. Then the equation of motion reads [27]

$$
\frac{\mathrm{d}(\gamma \beta)}{\mathrm{d} t}=\frac{2 R I}{\rho l c^{2}}\left(\frac{1-\beta}{1+\beta}\right)
$$

where $\rho$ is the target density, $\beta=v / c$ the target velocity, $\gamma=\left(1-\beta^{2}\right)^{-1 / 2}$ the Lorentz factor, and $R$ the reflectivity. The reflectivity can be approximated with the help of the normalized areal density $\sigma=\left(n_{e} / n_{c}\right) \times$ $\left(l / \lambda_{0}\right)$ by $R=1 /\left(1+\sigma^{-2}\right)$ [28]. The final velocity of the target $\beta_{f}$ can be derived as a function of the fluence $\mathcal{E}$ for a constant laser intensity [29]: 


$$
\beta_{f}=\frac{\left(1+\mathcal{E}-\sigma^{-2}\right)^{2}\left[1+\sqrt{1+4 \sigma^{-2}\left(1+\mathcal{E}-\sigma^{-2}\right)^{-2}}\right]+2 \sigma^{-2}-2}{\left(1+\mathcal{E}-\sigma^{-2}\right)^{2}\left[1+\sqrt{1+4 \sigma^{-2}\left(1+\mathcal{E}-\sigma^{-2}\right)^{-2}}\right]+2 \sigma^{-2}+2}, \quad \mathcal{E}=\pi \frac{m_{e}}{m_{p}} \frac{a_{0}^{2}}{\sigma} \frac{t}{T}
$$

with the period of the laser radiation $T$. This gives rise to a final kinetic energy of the target $T=\left(\gamma_{f}-1\right) m_{i} c^{2} \simeq$ $2 \mathrm{MeV}$ if we introduce the measured optimum thickness $l=25 \mathrm{~nm}$ which is in excellent agreement with the experimental result of $1.8 \mathrm{MeV}$. If the target was significantly thicker than the collisionless skin depth [Fig. 1(d)], the spectral shape of the protons exhibits a small peak at $2 \mathrm{MeV}$, corresponding to the velocity $\beta_{f}$, followed by a plateau region that is expected to extend down to the peak of the carbon spectrum at $\beta_{f} / 2$. Here, $\beta_{f} / 2$ is equal to the recession velocity of the plasma surface that is driven by the laser radiation pressure and commonly referred to as hole-boring velocity $[11,30,31]$. In the case of a target with a thickness below the optimum [Fig. 1(b)], the energy gap between the maxima of proton and carbon ions increases to $\sim 1 \mathrm{MeV}$ before the interface breaks apart and the carbon ions penetrate the proton layer exhibiting strong spectral modulations [Fig. 1(a)]. The gap is reduced to a minimum at the particular optimum target thickness [Fig. 1(c)]. This trend was also observed with a laser pulse duration of $70 \mathrm{fs}$ [Figs. 1(e)-1(h)]: The maximum carbon ion energy approaches a minimum in the proton energy distribution when reducing the target thickness to $15 \mathrm{~nm}$ [Fig. 1(e)].

Further, radiochromic films were used as spatial resolved detectors to determine the divergence of the accelerated ion beams. We obtained a total divergence of $140 \mathrm{mrad}$ at an energy per atomic mass unit of
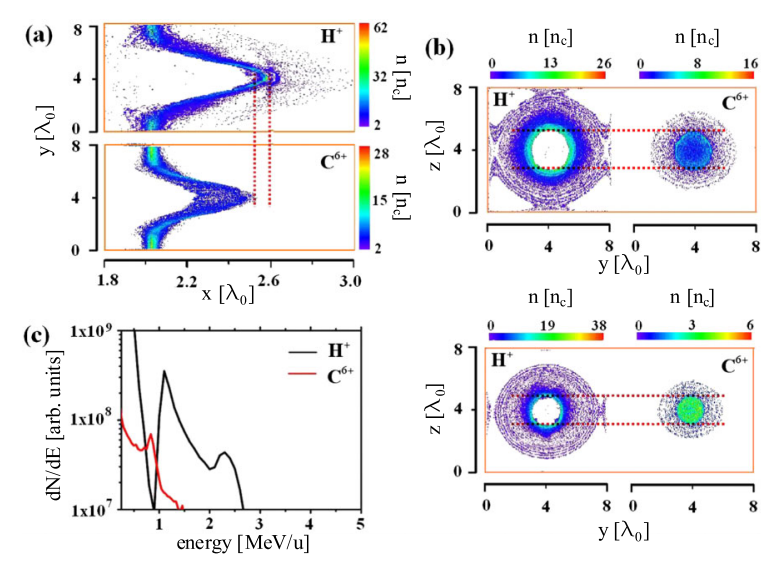

FIG. 2. Data from 3D-PIC simulation. (a) Spatial proton and carbon ion density distribution in the $x-y$ plane at $z=4 \lambda_{0}$ and $t=54 \mathrm{fs}$ after the arrival of the laser pulse. Obviously, the protons move ahead of the carbon ions. (b) Proton and carbon ion density distribution in the $y-z$ plane at $x=2.3 \lambda_{0}$ (top) and $x=2.4 \lambda_{0}$ (below) at the same time to indicate that the protons are always located outside of the carbon ion distribution. (c) Spectral distribution of protons (black) and carbon ions (red). The dashed red line in (a) and (b) illustrates the relative position of the proton with respect to the carbon ions. $\sim 1.4 \mathrm{MeV}$. We deduce a laser-to-ion-energy conversion efficiency by deconvolving the spectra shown in Fig. 1(c) in the region of the particular peak for protons (1.5-3 MeV) and fully ionized carbon (1.3-1.7 MeV/u) on the order of $0.5 \%$ and $6 \%$, respectively. The overall efficiency $(6.5 \%)$ is comparable with the heuristic light sail efficiency [13] $\eta=2 \beta_{f} /\left(1+\beta_{f}\right) \approx 12 \%$.

We support the experimental findings and simple analytical argumentation with PIC simulations in three (VLPL, [32,33]) and two (PSC, [34]) dimensions. The laser pulse was modeled with a Gaussian shape both in space and time with a duration of $35 \mathrm{fs}$ (FWHM), a spot size on the target of $4 \mu \mathrm{m}$ and a wavelength of $800 \mathrm{~nm}$, corresponding to $a_{0}=5$. The calculations in 3D are performed in a box with size of $4 \lambda_{0} \times 8 \lambda_{0} \times 8 \lambda_{0}$, sampled by $4000 \times 400 \times 400$ cells with 27 particles in each cell. The target was initiated at $x=2 \lambda_{0}$ as a flat foil with a thickness of $23 \mathrm{~nm}$ composed by the fully ionized carbon $\mathrm{C}^{6+}$ and hydrogen $\mathrm{H}^{+}$ions with particle number densities of $n_{\mathrm{C}^{6+}}=30 n_{c}$ and $n_{\mathrm{H}^{+}}=60 n_{c}$ corresponding to a plasma electron density of $n_{e}=240 n_{c}$. The dynamics show cylindrical symmetry as expected for a circularly polarized laser pulse. Figure 2(a) shows the proton and carbon density in the $x-y$ plane 54 fs after the impact of the peak intensity on the target. The protons have spatially separated from the heavier carbon ions due to their higher charge-to-mass ratio and therefore higher acceleration in the electric fields setup inside the target. Figure 2(b) shows the corresponding ion densities in two planes perpendicular to the laser propagation. Indeed, the separation of the two species is observed not only in the central region where the intensity is highest, but in the complete area that is irradiated by the laser pulse with proton number densities up to $30 n_{c}$. The spectral distribution of the ions that are accelerated close to the direction of the propagating laser pulse reveals very similar properties to the experimentally measured distributions [Fig. 2(c)]. To study the evolution in more detail, 2D simulations have been performed with the same laser and target initializations as in the 3D case and a simulation box size of $(15 \times 15) \mu \mathrm{m}^{2}$ sampled by $15000 \times 4000$ cells with 30 particles per cell. The target was initially situated at $x=7.5 \mu \mathrm{m}$. Figures 3(a)-3(d) show the evolution of the accelerated double layer plasma bunch constituting carbon ions and protons for times up to $223 \mathrm{fs}$, much longer than the laser pulse duration. All times are given with respect to the arrival of the maximum of the laser pulse at the target front side, where $t=0$. Indeed, the hydrogen and heavier carbon ions are separated at the very beginning of the interaction and a sharp interface between them is created $7 \mathrm{fs}$ after the arrival of the laser pulse maximum. This fast separation is best observed by the 

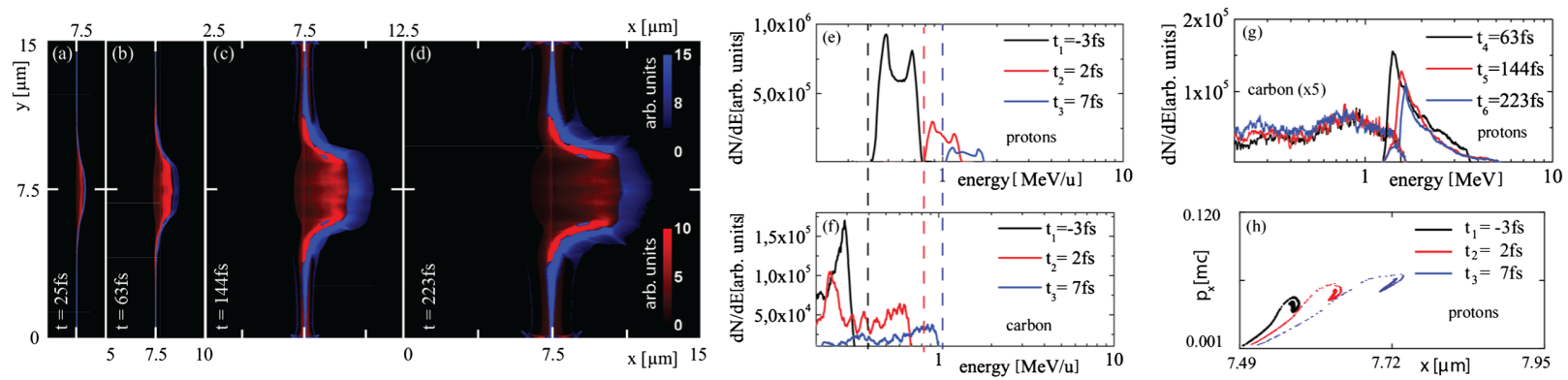

FIG. 3. Data from 2D-PIC simulation. (a)-(d) Spatial proton (blue) and carbon (red) ion density distribution in the $x$-y plane at different times (given in the figure caption) after the arrival of the peak of the Gaussian (in space and time) laser pulse. The spectral evolution during the laser-target interaction of protons (e), carbon ions (f), and after the interaction (g). In (h) the proton phase space during the interaction is revealing the characteristic spiral structure for RPA.

evolution of the ion spectra in Figs. 3(e) and 3(f) where particles accelerated close to the direction of the laser (0.03 rad cone angle) are taken into account. During this acceleration phase, the protons curl up in the phase space [Fig. 3(h)], representing a typical characteristic of radiation pressure acceleration. At later times, the instabilities initiated by the radiation pressure acting on the plasma develop into strong modulations of the carbon density as shown by the red color map in Figs. 3(a)-3(d). The protons, however, are pushed forward by the expanding carbon ion plasma [35] while the fastest carbon ions are hindered to penetrate the proton bunch so that the stable interface is preserved. This double layer is extremely stable and the corresponding energy distributions of protons and carbon ions in Fig. 3(g) show no substantial change after the interaction with the laser pulse. The obtained energies of the characteristic interface at $1.7 \mathrm{MeV} / \mathrm{u}$ and the isolated proton distribution centered around $1.9 \mathrm{MeV}$ [Fig. 3(g)] agree well with both our experimental observation and the analytical one-dimensional estimates from the light sail model [Eq. (1)].

In summary, we have presented an experimental study of the radiation pressure acceleration of ions with circularly polarized laser pulses and superb temporal contrast. A stable double layer composed from the target constituents, i.e., protons and carbon ions, has been generated with an overall laser-to-ion energy conversion of $6.5 \%$. The intensity dependence of the target thickness optimum and the achieved ion energies are in excellent agreement with the analytical 1D light sail model and are supported by realistic 2D- and 3D-PIC simulations. Our results indicate that dense ion bunches with energies of $1 \mathrm{GeV}$ can be generated with laser pulses focused onto $300 \mathrm{~nm}$ thick foils at an intensity of $\sim 2 \times 10^{22} \mathrm{~W} \mathrm{~cm}^{-2}$. Such targets are very robust and can be provided in high quantities allowing a high repetition rate, constituting a very attractive source for numerous applications. On the other hand, our results demonstrate that the laser pulse contrast has to be controlled carefully, i.e., it has to be assured that the laser intensity does not reach the damage threshold of the target earlier than 1 ps before the arrival of the main laser pulse which is a great challenge for future laser development. Furthermore, the observed stability of the double layer and its high ion densities over a wide range of parameters encourages a staged acceleration scheme using multiple laser pulses in order to enhance the ion energies while maintaining their monochromaticity [36].

\section{ACKNOWLEDGMENTS}

This work was partly supported by Deutsche Forschungsgemeinschaft through Transregio TR18 and the DFG-Cluster of Excellence Munich-Centre for Advanced Photonics (MAP). A. A. A. acknowledges the provided computation resources of JSC at project HBUIS.

[1] A. Henig et al., Phys. Rev. Lett. 103, 045002 (2009).

[2] A. Henig et al., Phys. Rev. Lett. 103, 245003 (2009).

[3] R. A. Loch, A. Lévy, T. Ceccotti, F. Quéré, C. Thaury, H. George, F. Bijkerk, K.-J. Boller, and Ph. Martin, Eur. Phys. J. Special Topics 175, 133 (2009).

[4] S. Steinke et al., Laser Part. Beams 28, 215 (2010).

[5] F. Dollar et al., Phys. Rev. Lett. 107, 065003 (2011).

[6] B. M. Hegelich et al., Nucl. Fusion 51, 083011 (2011).

[7] T. Esirkepov, M. Borghesi, S. Bulanov, G. Mourou, and T. Tajima, Phys. Rev. Lett. 92, 175003 (2004).

[8] A. Macchi, F. Cattani, T. Liseykina, and F. Cornolti, Phys. Rev. Lett. 94, 165003 (2005).

[9] X. Zhang, B. Shen, X. Li, Z. Jin, and F. Wang, Phys. Plasmas 14, 073101 (2007).

[10] O. Klimo et al., Phys. Rev. ST Accel. Beams 11, 031301 (2008).

[11] A. P. L. Robinson, M. Zepf, S. Kar, R. G. Evans, and C. Bellei, New J. Phys. 10, 013021 (2008).

[12] X. Q. Yan, C. Lin, Z. Sheng, Z. Guo, B. Liu, Y. Lu, J. Fang, and J. Chen, Phys. Rev. Lett. 100, 135003 (2008).

[13] A. Macchi, S. Veghini, and F. Pegoraro, Phys. Rev. Lett. 103, 085003 (2009).

[14] B. Qiao, M. Zepf, M. Borghesi, and M. Geissler, Phys. Rev. Lett. 102, 145002 (2009). 
[15] B. Qiao, M. Zepf, M. Borghesi, B. Dromey, M. Geissler, A. Karmakar, and P. Gibbon, Phys. Rev. Lett. 105, 155002 (2010).

[16] T.-P. Yu, A. Pukhov, G. Shvets, and M. Chen, Phys. Rev. Lett. 105, 065002 (2010).

[17] S. V. Bulanov, E. Yu. Echkina, T.Zh. Esirkepov, I. N. Inovenkov, M. Kando, F. Pegoraro, and G. Korn, Phys. Rev. Lett. 104, 135003 (2010).

[18] A. Jullien et al., Opt. Lett. 30, 920 (2005).

[19] M. Kalashnikov et al., in Proceeding of the CLEO Science \& Innovations 2011, Baltimore, Maryland (Optical Society of America, Washington, DC, 2011) [http:// www.opticsinfobase.org/abstract.cfm?uri=CLEO:\%20S\% 20and\%20I-2011-CWG3].

[20] A. Lévy et al., Opt. Lett. 32, 310 (2007).

[21] A. A. Andreev, S. Steinke, T. Sokollik, M. Schnurer, S. Ter Avetsiyan, K. Yu. Platonov, and P. V. Nickles, Phys. Plasmas 16, 013103 (2009).

[22] M. Allen, P. Patel, A. Mackinnon, D. Price, S. Wilks, and E. Morse, Phys. Rev. Lett. 93, 265004 (2004).

[23] S. Ter-Avetisyan, M. Schnürer, and P. V. Nickles, J. Phys. D 38, 863 (2005).
[24] X. Q. Yan et al., Appl. Phys. B 98, 711 (2010).

[25] V. K. Tripathi, C. S. Liu, X. Shao, and A. Sharma, Phys. Plasmas 18, 043103 (2011).

[26] C. A. J. Palmer et al., Phys. Rev. Lett. 108, 225002 (2012).

[27] J. F. L. Simmons and C. R. McInnes, Am. J. Phys. 61, 205 (1993).

[28] V. A. Vshivkov, N. M. Naumova, F. Pegoraro, and S. V. Bulanov, Phys. Plasmas 5, 2727 (1998).

[29] A. Macchi, S. Veghini, T. V. Liseykina, and F. Pegoraro, New J. Phys. 12, 045013 (2010).

[30] S. C. Wilks, W. Kruer, M. Tabak, and A. Langdon, Phys. Rev. Lett. 69, 1383 (1992).

[31] A. Macchi and C. Benedetti, Nucl. Instrum. Methods Phys. Res., Sect. A 620, 41 (2010).

[32] A. Pukhov, J. Plasma Phys. 61, 425 (1999).

[33] T.P. Yu, A. Pukhov, G. Shvets, M. Chen, T. H. Ratliff, S. A. Yi, and V. Khudik, Phys. Plasmas 18, 043110 (2011).

[34] A.J. Kemp and H. Ruhl, Phys. Plasmas 12, 033105 (2005).

[35] S. S. Bulanov et al., Phys. Rev. E 78, 026412 (2008).

[36] Z. M. Zhang, X. T. He, Z. M. Sheng, and M. Y. Yu, Phys. Plasmas 18, 023110 (2011). 